\title{
CONTRIBUIÇÕES PARA UMA ARQUEOLOGIA DE ROMA PÓS-COLONIAL: LIGANDO BRASIL E GRÃ-BRETANHA*
}

\author{
Richard Hingley ${ }^{1}$
}

\section{Conexões entrelaçadas}

Esse artigo revisa minhas colaborações junto ao Professor Funari a fim de fornecer um enfoque pessoal sobre a significativa contribuição que ele fez ao campo de teoria e arqueologia histórica. $O$ foco desta minha contribuição está sobre o período romano, o qual consiste em apenas uma parte do amplo rol de interesses de Funari. Meu artigo está baseado, particularmente, nos vínculos que eu e esse professor estabelecemos desde 1999. Meu primeiro contato com Funari se deu quando contribuí para um livro que ele coeditou com Martin Hall e Sîan Jones, Historical Archaeology: Back from the Edge (Funari, Hall and Jones Eds, 1999). Esse volume provém de uma sessão de conferências realizadas no âmbito do terceiro World Archaeology Congress 3 em Nova Deli, Índia, no ano de 1994. Infelizmente eu não pude participar, apesar disso Sîan Jones me convidou para contribuir com um artigo para a publicação. Meu texto ressaltou algumas abordagens críticas à Britânia Romana que objetivavam afastar o enfoque dos estudos da elite e das populações militares da província (Hingley 1999). Ele se baseava em argumentos que eu já ressaltara em meu livro Rural Settlement in Roman Britain (Hingley, 1989).

Foi apenas em 1999 que tomei conhecimento, pela primeira vez, do amplo interesse acadêmico e das contribuições para os estudos de Arqueologia de Roma de Funari. Minha lembrança é de que, nesse ano, nos encontramos no World Archaeology Congress 4 na Cidade do Cabo, na África do Sul. Nesse congresso minha comunicação abordava os sítios de abandono das Terras Altas e da Ilha da Escócia (Hingley, 2000a). Minha pesquisa sobre os sítios de abandono da Escócia objetivou priorizar os resquícios arqueológicos das comunidades desapropriadas que foram expulsas de suas terras para abrir o caminho para produção de ovelhas; muitas dessas pessoas foram forçadas a emigrar para os Estados Unidos e

\footnotetext{
* Texto traduzido do inglês por Alexandre Cozer, doutorando em História, Universidade Federal do Paraná.

1 Professor Doutor, Universidade de Durham, Durham, Reino Unido. email: richard.hingley@durham.ac.uk
} 
para a Austrália. No começo de 1999 eu trabalhava como um Inspector of Ancient Monuments para a Historic Scotland 2, o serviço de patrimônio do Estado. O fornecimento de patrimônio na Escócia priorizara os resquícios tangíveis dos fortes e fronteiras romanos, castelos medievais e conventos. Sítios de abandono pareceram-me fornecer uma narrativa alternativa que abordasse o desapropriado. A ênfase principal de minha pesquisa, entretanto, recaiu sobre o período romano.

No outono de 1999 passei a ocupar o posto de Professor de Arqueologia Romana na Universidade de Durham (Inglaterra). Como um resultado disso, meu contato com Funari aumentou, particularmente após a publicação de meu livro Roman Officers and English Gentlemen (2000b). Esse livro explorou a origem colonial da Arqueologia de Roma na GrãBretanha, abordando as pesquisas de historiadores e arqueólogos da Antiguidade entre os finais do século dezenove e princípios do século vinte. Ainda editei um volume separado intitulado Images of Rome 3 (2001), o qual continha uma coleção de artigos; esses textos abordavam o modo como o passado do Império Romano tem sido aproveitado em vários países europeus e nos Estados Unidos da América (Hingley ed., 2001). Funari resenhou esses artigos e acredito também que ele percebia a relevância deles para o contexto das abordagens pós-coloniais que ele tentava promover dentro da arqueologia brasileira. Ele organizou para que o artigo que eu havia publicado em Images of Rome fosse traduzido para o português brasileiro e publicado nos Textos Didáticos número 47 (Hingley, 2002). Esse volume também incluía um artigo de Jean-Pierre Vernant intitulado "Fronteiras do Mito" a uma introdução feita por Renata Senna Garraffoni ${ }^{4}$. Eu me lembro de ter ficado extremamente lisonjeado pelo meu artigo sobre os usos das "Imagens de Roma" na Inglaterra ter tido relevância para arqueólogos no Brasil, o que também ajudou a me encorajar a dar sequência às minhas investigações póscoloniais.

Nossos primeiros debates indicaram que eu e Funari tínhamos muitos interesses em comum e então organizamos a sessão "Colonialism and Identity: Origins and Otherness", no World Archaeology Congress 5, em Washington em 2003. Essa foi uma sessão substancial e calorosa e,

\footnotetext{
2 A tradução literal do título do cargo seria "inspetor de monumentos antigos". (Nota do Tradutor)

${ }^{3} \mathrm{O}$ título significa "Imagens de Roma" e representa também o interesse do autor pelos usos que foram dados ao passado romano na Inglaterra e no mundo. Na sequência do texto, esse título volta a ser utilizado como um conceito e, portanto, o mencionaremos como "Imagens de Roma", seguindo a tradução feita por Garraffoni na coleção didática mencionada por Hingley. (Nota do Tradutor)

4 Textos Didáticos n 47 foi republicado em uma versão expandida em 2005.
} 
particularmente, me lembro do debate sobre os mitos de origem e o colonialismo. Quando descobri, no Congresso, o quanto Funari estava atarefado com seu envolvimento no movimento World Archaeology, sentime profundamente agradecido por ele ter encontrado tempo para ajudar a organizar nossa sessão e contribuir com ela. Ao olhar a lista dos contribuintes com a sessão, percebi o nome de um dos estudantes de graduação de Funari, Renato Pinto, quem eu ainda voltaria a encontrar no Brasil. Funari e eu discutimos publicar os resultados dessa sessão, mas estávamos ambos ocupados demais para dar sequência à ideia.

Esses contatos com Funari ajudaram a desenvolver meus interesses pela teoria pós-colonial e pelo Imperialismo Romano. As três sessões sucessivas com as quais contribuí dentro do World Archaeology Congress me permitiram comunicar os argumentos que vinha desenvolvendo para uma plateia muito mais ampla do que aquela formada por meus colegas e amigos no âmbito do desenvolvimento do movimento de arqueologia pós-colonial na Europa. Me encontrava particularmente atraído pelo trabalho acadêmico de Funari devido ao seu percurso pela Arqueologia de Roma, incluso sua pesquisa de pós-doutorado sobre as ânforas romanas (Funari 1996), mas sobretudo devido ao seu interesse em arqueologia histórica, imperialismo, colonialismo e patrimônio interesses que compartilhávamos.

À medida que minha pesquisa se desenvolveu, perdi algum contato com o World Archaeology Congress mas os três com os quais contribuí me permitiram estender meus contatos internacionais, bem como fundar a pesquisa que eu empreendia em um contexto teórico mais amplo. Em 2007 Funari me pedi que escrevesse um artigo para um volume editado de New Perspectives on the Ancient World, que ele produzia com dois colegas (Funari, Garraffoni e Latalien eds., 2008). Esse livro aborda as "percepções modernas e representações antigas" e, novamente, eu me sentia lisonjeado por contribuir com um artigo, dado que a recepção histórica da cultura romana e o imperialismo são objetos que ocupam um lugar central da minha pesquisa desde os finais dos anos 1990. Essa obra fornece uma perspectiva internacional sobre as formas como o passado clássico tem sido empregado ao longo do mundo. Apesar de Funari ter um largo rol de artigos e livros que abordam uma variedade de questões da política da arqueologia histórica, essa obra também logra ressaltar sua significante contribuição para a arqueologia do período romano. Juntamente com outros livros editado que Funari produziu (Funari, Jones Hall eds, 1999; Funari, Zarankin and Stobel Eds, 2005), essa obra também ilustra a extensão da rede de colegas, de colaboradores internacionais e de alunos que tem esse pesquisador. 
Minha carreira acadêmica na Universidade de Durham me proporcionou desenvolver pesquisas sobre a Arqueologia de Roma associada à busca de uma perspectiva pós-colonial e historiográfica e em 2005 eu publiquei Globalizing Roman Culture (Hingley, 2005). Meus contatos com Funari e seus estudantes permaneceram muito importantes ao passo que minha pesquisa se desenvolvia. Fiquei extremamente honrado quando Funari me convidou para ir ao Brasil para uma série de palestras em Agosto de 2008, a qual foi composta diversas palestras em Campinas e uma em Curitiba, onde fui recebido por Renata Senna Garraffoni. Minha parceira e musa intelectual, Christina Unwin, pode viajar comigo e compartilhamos ótimos momentos com Funari e seus orientandos - o que foi uma excelente ocasião social tanto para mim quanto para Christina, além de um evento muito estimulante. Me lembro das discussões acadêmicas acaloradas e muito me impressionou a grande importância que a arqueologia tinha para o muitos daqueles para os quais falei no Brasil - o que se deu, ao meu ver, devido a ênfase de grande parte da pesquisa tanto do Funari quanto de seus alunos repousar sobre os temas do patrimônio e da importância presente do passado romano. $\mathrm{O}$ patrimônio nos coloca em contato direto com a relevância contemporânea da arqueologia. Na tradição intelectual da Arqueologia de Roma os acadêmicos tenderam a enfatizar uma percepção distanciada, o que ofusca o contexto cultural e político no qual ideias são geradas e consumidas - eu tentei desafiar essa pauta em minha pesquisa (Hingley, 2015a). Para mim, a maior relevância da pesquisa pós-colonial e sobre o patrimônio que se desenvolveu no Brasil está no enfoque dado à importância contemporânea da investigação arqueológica. E minha estadia no Brasil impactou profundamente minha própria pesquisa.

No começo dos anos 2000 eu já começava a me voltar às questões da importância contemporânea do passado romano. Meu objetivo inicial se concentrara na crítica da teoria da Romanização (Hingley, 2000b; Hingley, 2001). Entre os finais dos anos 1990 e os anos iniciais do novo milênio um grupo de arqueólogos da Inglaterra, incluso David Mattingly e Jane Webster, estavam empenhados em uma crítica pormenorizada da teoria da Romanização e minha pesquisa fazia parte desse movimento pós-colonial. $\mathrm{O}$ artigo que Funari publicara nos Textos didáticos $n^{\circ} 47$ abordava esse tema - enfocava os usos do passado romano nas sociedades Vitoriana e Eduardiana na Grã-Bretanha. Meus interesses, entretanto, estavam mudando e o livro Globalizing Roman Culture (2005) usou da teoria pós-colonial para explorar o modo como o Império Romano havia sido recriado no mundo contemporâneo, um tema que continua a me fascinar (Hingley 2015a). Minhas palestras em Campinas e em Curitiba em 2008 deram sequência a essa preocupação com 
explorarem o contexto político por detrás de teorias contemporâneas da identidade romana e das mudanças culturais. No Brasil eu também pude delinear minha pesquisa inicial sobre a vida póstuma da Muralha de Adriano, o principal monumento romano da Britânia; posteriormente publiquei essa pesquisa em Hadrian's Wall: A Life (Hingley, 2012).

Funari e seus colegas publicaram uma coleção de artigos meus traduzidos para o português brasileiro como um resultado da minha visita ao país (Hingley 2010), incluindo ali textos que eu publicara em 1991, 1996 e 2008. Uma introdução abordando a teoria e os estudos romanos no Brasil foi feita a esses artigos por Renata Garraffoni, Pedro Funari e Renato Pinto. Eu retornei muito brevemente ao país em 2009 para uma comunicação em um festival literário e, novamente, o agradável convite vinha de Funari. É uma pena que não tenha podido retornar recentemente ao Brasil devido, principalmente, a outros compromissos.

Em 2013 eu e Funari escrevemos artigos separados para uma sessão de um congresso na Sociedade pela Arqueologia Histórica (Society for Historical Archaeology) ocorrido em Leicester (Reino Unido) e organizado por Craig Cipolla e Katherine Hayes. Essa sessão foi posteriormente publicada como um livro intitulado Rethinking Colonialism (Cipolla e Hayes eds., 2015). O artigo de Funari, que foi escrito em colaboração com Lúcio Menezes Ferreira, relaciona a escravidão Antiga e Moderna (Ferreira e Funari, 2005). Meu artigo explorou a ideia de identificar "Comunidades Descendentes" no estudo da Britânia Romana (Hingley, 2015b). De algum modo, havia me distanciado do campo da arqueologia histórica nos cinco anos anteriores, mas as acaloradas discussões desse congresso atraíram novamente minha atenção para o potencial das comparações interculturais e intertemporais. Meu artigo para o livro editado me permitiu esboçar uma nova iniciativa que estava planejando e que, posteriormente, tornou-se o projeto "Ancient Identities" (Bonacchi, Hingley e Yarrow, 2016). Esse projeto - o qual envolve também meus colegas Chiara Bonacchi, Tom Yarrow e Kate Sharpe explora o uso de ideias extraídas da Idade do Ferro e do passado romano pelas partes interessadas ${ }^{5}$ no patrimônio antigo ao longo da Inglaterra da Escócia e do País de Gales ${ }^{6}$, e procura perceber esses exemplos de casos

5 "partes interessadas" é a tradução que propomos ao termo em inglês stakeholder. É também a tradução literal do termo. Na Arqueologia que se preocupa com os usos do patrimônio e do passado no presente, esse termo designa as diversas instituições ou grupos que podem estar interessados no passado por algum motivo. Na falta de um termo tão abrangente em português, optamos por explicar a escolha de uma tradução literal. (Nota do Tradutor)

${ }^{6}$ Essa pesquisa é financiada pela Arts and Humanities Research Council pelos anos entre 2016 e 2018. 
em um contexto internacional amis amplo. Tal projeto marca o fim de uma transição no meu trabalho que começou com estudo dos usos das "Imagens de Roma" no contexto Vitoriano e Eduardiano e agora transitou para o estudo da recepção da Idade do Ferro e do passado romano no presente. $\mathrm{O}$ vínculo com Funari e seus colaboradores, nesse ponto, é direto, uma vez que suas pesquisas auxiliaram a me inspirar a mudar de enfoque.

\section{Transformando objetivos}

Um dos principais textos que usamos para desenvolver o projeto "Ancient Identities" (identidades antigas) foi "The Uses of Roman Heritage in Brazil" por Pedro Funari e Renata Garraffoni (2012). Esse é um dos poucos trabalhos que encontrei que aborda explicitamente a importância presente do patrimônio romano, um tema que é extremamente ausente do campo teórico dos estudos de Arqueologia de Roma na Grã-Bretanha. No Brasil, uma abordagem crítica se desenvolveu na última década e meia, a qual debate o uso do patrimônio romano na academia e na cultura popular (ibid., 53). Essa pesquisa explorou a relação entre patrimônio, identidade moderna, conceitos e materiais definidos a partir passado romano a fim de estabelecer o lugar do Brasil na cultura ocidental. Apesar de haver algumas conexões entre as pesquisas que vêm se desenvolvendo no Brasil e a Arqueologia de Roma Pós-Colonial que tem se desenvolvido na Grã-Bretanha (Garraffoni, Funari e Pinto, 2010), os estudos no Brasil têm uma trajetória de desenvolvimento diversa e têm um vínculo mais estreito com os argumentos e debates desenvolvidos no interior do World Archaeology Congress durante os finais dos anos 1990.

A própria pesquisa de Funari aborda o contexto colonial e nacional no qual os modelos e ideias clássicos foram recebidos no Brasil. Esse trabalho se desenvolveu juntamente com a crítica da Romanização empreendida na Inglaterra. Funari explorou os vínculos estreitos que existiram entre o colonialismo e as formas opressoras de governo que houveram no Brasil até a década 1980 (Ferreira e Funari 2015). Ele também estudou o modo como a cultura clássica foi adotada e manipulada no Brasil durante o período colonial, e até mesmo os posteriores usos de Roma como um modelo para o Império do Brasil (Garraffoni e Funari 2012, 58). O estudo dessas conexões entre o passado e o presente é o motivo pelo qual a teoria pós-colonial foi introduzida ao estudo arqueológico no Brasil (ibid., 66-7). Um enfoque mais recente desses estudos aborda o significado do patrimônio romano para a 
população do presente, desenvolvendo um campo de estudos que tem um grande valor para sociedade que vem se estabelecendo no Brasil desde que a ditadura foi substituída pela democracia em 1985 (ibid., 71). Essa preocupação com as identidades dos brasileiros contemporâneos ajudou a estimular minha própria pesquisa em andamento à época sobre o uso das "Imagens de Roma", estendendo-a para o presente europeu e levando ao desenvolvimento do projeto "Ancient Identities".

O passado romano é percebido, no Brasil, como detentor do potencial de incentivar a diversidade e a justiça social. Um exemplo que Garraffoni e Funari (ibid., 68-70) fornecem envolve os modelos criados pelo estudo arqueológico de Pompeia, os quais são usados como comparativo para questões sociais inerentes da sociedade brasileira atual, tais como aquelas relacionadas com a identidade de pessoas comuns, com a diversidade, com relações de gênero e sexualidade. Garraffoni e Funari (ibid., 70) argumentam que:

De um diálogo com os estudiosos europeus que buscam criar interpretações menos estáticas do mundo romano, alguns estudiosos brasileiros têm percebido que a arqueologia permite a construção de modelos teóricos menos excludentes. Tal posição política é fundamental não apenas para se questionar abordagens acadêmicas tradicionais e a recepção moderna do passado romano baseada no poder e em formas excludentes de identidade nacional, mas também para se pensar outras formas de sensibilidades e de visões de mundo.

Essa adoção de uma abordagem pós-colonial na Arqueologia de Roma vai além da desconstrução de discursos nacionalistas e colonialistas, enfatizando também que as interpretações do passado podem relacionarse diretamente com a relevância que esse passado tem para as sociedades e as partes interessadas do presente. Isso sugere que a nova pesquisa sobre o Império Romano pode ser pensada e comunicada para as partes interessadas a fim de criar comparações e contrastes sem que se perca de vista questões de poder que estão emaranhadas nas complexas relações que ligam o passado ao presente (Hingley, 2015a).

Atualmente, a mais interessante comparação com o trabalho sobre a inclusão social no Brasil é a pesquisa no Reino Unido que tem se debruçado sobre as mobilidades e a diáspora no período romano (Eckardt et al., 2014; Eckardt e Müldner, 2016). O estudo de ossos humanos por meio da análise de isótopos estáveis e de aDNA (ADN antigo) tem demonstrado a consideravelmente intensa migração que ocorreu para a Britânia dentre as populações que vivam nos principais centros urbanos como Londres, York e Winchester. Arqueólogos e educadores têm empreendido um esforço consciente para usar esse material a fim de argumentar que a imigração para a Britânia não é um 
fenômeno recente. Isso faz parte de um empenho em tornar as pessoas mais tolerantes com os seus próximos em uma época que é caracterizada pelo aumento do nacionalismo e do racismo. Nós acreditamos que a Idade do Ferro e o patrimônio romano têm um potencial considerável para abordar sensibilidades e visões de mundo atuais e nosso projeto "Ancient Identities" tem perseguido esse objetivo (Hingley, Bonacchi e Sharpe, no prelo).

Minha principal dívida com o Funari - além de sua grande generosidade e hospitalidade durante minhas duas idas ao Brasil - se deve ao fato de ele ter me ajudado a perceber, gradualmente, o significado do patrimônio no contexto da Arqueologia de Roma. Minhas viagens ao Brasil, minhas discussões com Funari, juntamente com os artigos que ele escreveu sobre o patrimônio romano, tiveram impacto direto ao me estimularem a me preocupar mais detidamente com a relevância contemporânea das "Imagens de Roma". Me sinto profundamente agradecido pela oportunidade que esse artigo me proporcionou de enfatizar a importância da liderança acadêmica de Pedro, de sua amizade e erudição.

\section{Bibliografia}

BONACCHI, C., R. HINGLEY and T. YARROW. 'Exploring Ancient Identities in Modern Britain', Archaeology International 19, 2016, 54-7. <http://doi.org/10.5334/ai.1909>.

CIPOLLA, C.N. and K.H. HAYES (eds). Rethinking Colonialism: Comparative Archaeological Approaches. Gainesville, Fl, University Press of Florida, 2015.

ECKHARDT, H. and G. MÜLDNER. Mobility, Migrations and Diasporas in Roman Britain', in M. MILLETT, L. REVELL and A. MOORE (eds) The Oxford Handbook of Roman Britain. Oxford, Oxford University Press, 2016, 203-223.

ECKHARDT, H., G. MÜLDNER and M. LEWIS. 'People on the move in Roman Britain', World Archaeology 46(4), 2014, 534-550.

FERREIRA, L.M. and P.P. FUNARI. The Archaeology of Slavery Resistance in Ancient and Modern Times: An initial outlook from a Brazilian perspective', in C. CIPOLLA and K.H. HAYES (eds), 2015, 190209. 
FUNARI, P.P. Dressel 20 inscriptions from Britain and the consumption of Spanish olive oil. Oxford, BAR British 250, 1996.

FUNARI, P.P., R. S. GARRAFFONI and B. LATIEN (eds). New Perspectives on the Ancient World: Modern Perceptions, Ancient Representations. Oxford, BAR International 1782, 2008.

FUNARI, P.P., M. HALL and S JONES (eds). Historical Archaeology: Back from the Edge. London, Routledge, 1999.

FUNARI, P.P., A. ZARANKIN and E. STOVEL (eds). Global Archaeological Theory: contextual voices and contemporary thoughts. New York/Boston, Kluwer, 2005.

GARRAFFONI, R. S. and P. P. FUNARI. 'The Uses of Roman Heritage in Brazil: Traditional Reception and new Critical Approaches,' Heritage $\mathcal{E}$ Society 5(1), 2012, 53-76.

GARRAFFONI, R.S., P.P. FUNARI and R. PINTO. 'O Estudo da Antiguidade no Brasil: as contribuiçōes das discussōes recentes', in R. HINGLEY, 2010, 9-25.

HINGLEY, R. 'The imperial context of Romano-British studies and proposals for a new understanding of social change', in P.P. FUNARI, M. HALL and S. JONES (eds), 1999, 137-49.

-- 'Medieval or Later Rural Settlement in Scotland: The Value of the Resource', in J. ATKINSON, I. BANKS and G. MacGREGOR (eds.) Townships to Farmstead: Rural Settlement Studies in England and Wales. BAR British Series 293, 2000a, 11-9.

-- Roman Officers and English Gentlemen. London: Routledge, 2000b.

-- (ed.). Images of Rome: Perceptions of ancient Rome in Europe and the United States of America in the modern age. Journal of Roman Archaeology, Supplementary Series no. 44, 2001.

-- Concepçōes de Roma: uma perspectiva inglesa', in textos Didáticos: repensando o mundo antigo 47, 2002, 27-62.

-- Globalizing Roman Culture: Unity, Diversity, Empire. London, Routledge, 2005.

-- O Imperialismo Romano: novas perspectivas a partir da Bretanha. Butantã, Annablume, 2010. 
-- Hadrian's Wall: A Life. Oxford, Oxford University Press, 2012.

-- 'Post-colonial and Global Rome: the genealogy of empire'. In M. PITTS and J. M. VERSLUYS (eds.) Globalisation and the Roman World: World History, Connectivity and Material Culture. Cambridge: Cambridge University Press, 2015a, 32-46.

-- 'Working with Descendant Communities in the Study of Roman Britain: Fragments of an Ethnographic Project Design'. In C.N. CIPOLLA and K.H. HAYES (eds.), 2015b, 161-89.

MATTINGLY, D. 'Dialogues of Power and Experience in the Roman Empire'. in D. MATTINGLY (ed.) Dialogues in Roman Imperialism: Power, discourse, and discrepant experiences in the Roman Empire. Portsmouth, Rhode Island, Journal of Roman Archaeology. No. 23, 1997, 7-25.

-- 'Being Roman: expressing identity in a provincial setting', Journal of Roman Archaeology 17, 2004, 5-25.

WEBSTER, J. 'Roman imperialism and the "post-imperial age"', in J. WEBSTER \& N. COOPER (eds) Roman Imperialism: Post-colonial perspectives. Leicester, School of Archaeological Studies, University of Leicester. No. 3, 1996, 1-18. 